\title{
La cinta muraria ed i fossati della città di Otranto. Il rilievo tridimensionale integrato per la conoscenza delle evidenze architettoniche ed archeologiche
}

The walls and moats of the city of Otranto. An integrated three-dimensional survey for studying the architectural and archaeological evidence

\author{
Carmine Mitello $^{\text {a }}$, Giovanna Muscatello ${ }^{\text {b }}$ \\ ${ }^{a}$ Musa S.r.l., Giurdignano (Le), Italy, c.mitello@tiscali.it \\ ${ }^{\text {b }}$ Dipartimento di Beni Culturali - Università del Salento, Lecce, Italy, giovannamuscatello@libero.it
}

\begin{abstract}
The system of fortifications that rings the old town of the city of Otranto (Puglia, Italy) conserves the still visible traces of structures belonging to various historical periods, attesting to the evolution of the settlement's defensive system from the Messapian period (fifth century BC) to the Aragonese period (sixteenth century). As part of a recent urban renewal project targeting the area of the moats, new archaeological, historic and architectural investigations were conducted. These included a painstaking analysis of the circuit of defensive walls and the broad and deep moat, which contains valuable archaeological and architectural evidence that has never before been studied. The use of advanced surveying technologies such as 3D Laser Scanners, parametric and georeferenced, enabled a holistic, synoptic and comparative reading of the structures, recording the distinctive features, building techniques, materials, alignments, range of thicknesses and losses of continuity in the walls, all of which are necessary for a correct identification of the construction phases. In addition, the data arising from the study and threedimensional survey of some subterranean tunnels, entirely excavated in the rock in the area of the moats surrounding the Aragonese castle, have also enriched the framework of knowledge regarding specific military and defensive dynamics.
\end{abstract}

Keywords: Otranto, laser scanner 3D, moats, subterranean tunnels.

\section{Introduzione}

Il circuito murario ed i fossati della città di Otranto, collocata lungo la costa ionica della Penisola Salentina (Puglia, Italia), custodiscono la storia del sistema di difesa del centro idruntino (Fig. 1). Nell'ambito di un recente progetto di riqualificazione dei fossati della città è stato possibile compiere uno studio analitico delle strutture, attuando un nuovo processo di conoscenza, ancora in corso, necessario ai fini di una complessiva valutazione delle evidenze storico- architettoniche ed archeologiche che contraddistinguono questo importante contesto monumentale, che comprende il circuito murario, l'ampio fossato, l'antemurale, il maestoso castello ed i camminamenti sotterranei.

Il rilievo metrico diretto e strumentale ha costituito una fase imprescindibile; un approccio conoscitivo all'opera costruttiva nella sua evoluzione diacronica. L'acquisizione accurata dei dati metrici e materici e l'elaborazione degli stessi 
con software dedicati sono state finalizzate alla costruzione di un modello tridimensionale che ha contribuito alla lettura architettonica e stratigrafica del complesso organismo oggetto di studio.

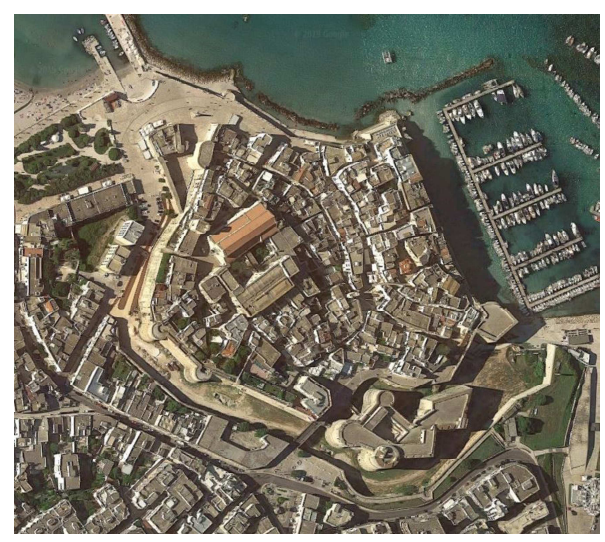

Fig. 1. Otranto, vista zenitale del centro storico con il circuito delle mura e i fossati (Google Maps).

\section{Il rilievo tridimensionale integrato: metodo di conoscenza}

Lo sviluppo delle tecnologie messe a disposizione nel campo della documentazione ha fornito ottime opportunità per il rilevamento metrico e per l'analisi tecnica del patrimonio culturale, in particolar modo nel settore dei beni architettonici e archeologici, sia per quanto riguarda l'acquisizione delle informazioni riferite al bene storico oggetto d'indagine, sia per quanto riguarda le molteplici possibilità offerte dai metodi di rappresentazione e di divulgazione. Al fine di una corretta metodologia di intervento, le operazioni di rilievo sono state strutturate e realizzate in diverse fasi: il rilievo topografico, come impalcatura geometrica di riferimento in cui individuare i punti di appoggio per i rilievi di dettaglio, il rilievo laser scanner 3D e le operazioni di post-processing. La rete topografica di impianto, con il posizionamento e la materializzazione dei vertici della poligonale, univocamente riconoscibili nei diversi momenti del rilievo, è stata impostata con l'uso di sistemi di posizionamento satellitari GNSS. Alla poligonale principale sono state collegate delle reti di raffittimento per le integrazioni topografiche effettuate con Total Station, rese necessarie per la realizzazione di rilievi di dettaglio sia all'esterno che all'interno dei sotterranei, unitamente alle prese acquisite con il laser scanner (Fig. 2).

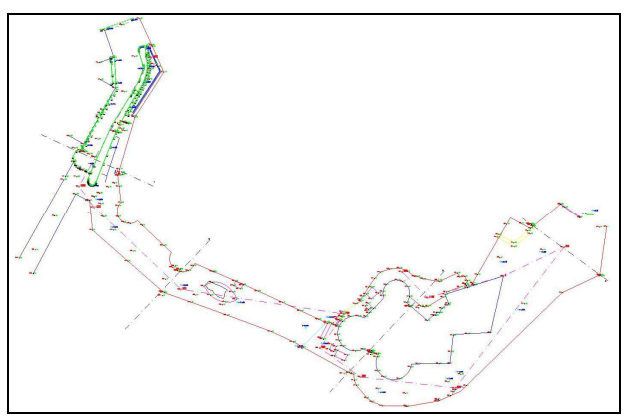

Fig. 2. Fossati. Rilievo topografico integrato.

L'esecuzione del rilievo integrato con tecnologia laser scanner 3D ad alta definizione (real-based) dell'intero percorso dei fossati, delle mura, dei camminamenti sotterranei e delle aree archeologiche, esplorate nell'ambito dei lavori di scavo archeologico, ha previsto una valutazione preliminare degli oggetti da rilevare e una preventiva verifica della visibilità e dell'accessibilità. In particolare, le strutture, le dimensioni e le relazioni spaziali, nello sviluppo orizzontale e in quello verticale, hanno inciso notevolmente sulle modalità di esecuzione del rilevamento; la campagna di rilievo si è svolta distribuendo sul campo una serie di stazioni successive, collegate visivamente tra loro al fine di acquisire la massima quantità di dati metrici ed evitare assenza di dati. L'utilizzo della tecnologia laser scanner 3D integrata a metodi fotogrammetrici e topografici ha consentito l'acquisizione di dati che descrivono la geometria dell'oggetto con un livello di accuratezza tale da registrare milioni di punti con precisione millimetrica (Figg. 3-5). Oltre all'acquisizione delle coordinate spaziali "x, y e z" di ogni singolo punto che compongono la geometria del complesso fortificato e delle aree circostanti, la strumentazione ha rilevato un quarto dato, la riflettanza, che rappresenta la quantità di luce restituita dal materiale indipendentemente dalla luce ambientale. In questo modo ogni superficie è stata descritta sia dal punto di vista quantitativo che qualitativo. 
Tale procedimento ha permesso l'elaborazione di un modello tridimensionale della fabbrica architettonica completamente misurabile che rappresenta un'insostituibile risorsa per chiarire gli aspetti relativi alle fasi storiche e alle tecniche costruttive impiegate.

\subsection{I fossati}

Il fossato rappresenta uno degli elementi costitutivi del sistema difensivo di Otranto, oltre ad allontanare la cittadella fortificata dal resto del territorio, potenziava la separazione del castello

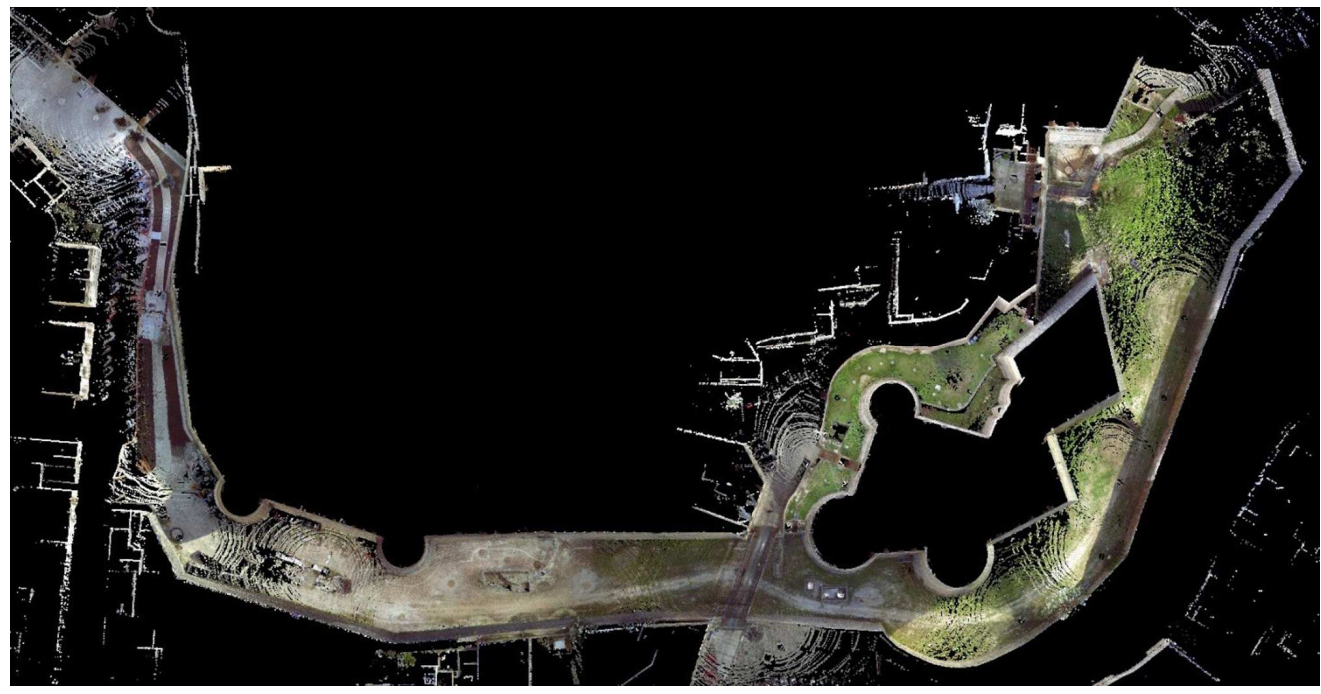

Fig. 3. Otranto. Rilievo laser scanner 3D .Vista dall'alto dei fossati che corrono lungo il perimetro del nucleo storico della città e del castello aragonese. In evidenza le torri e l'andamento del muro di controscarpa (Mitello \& Muscatello, 2018).

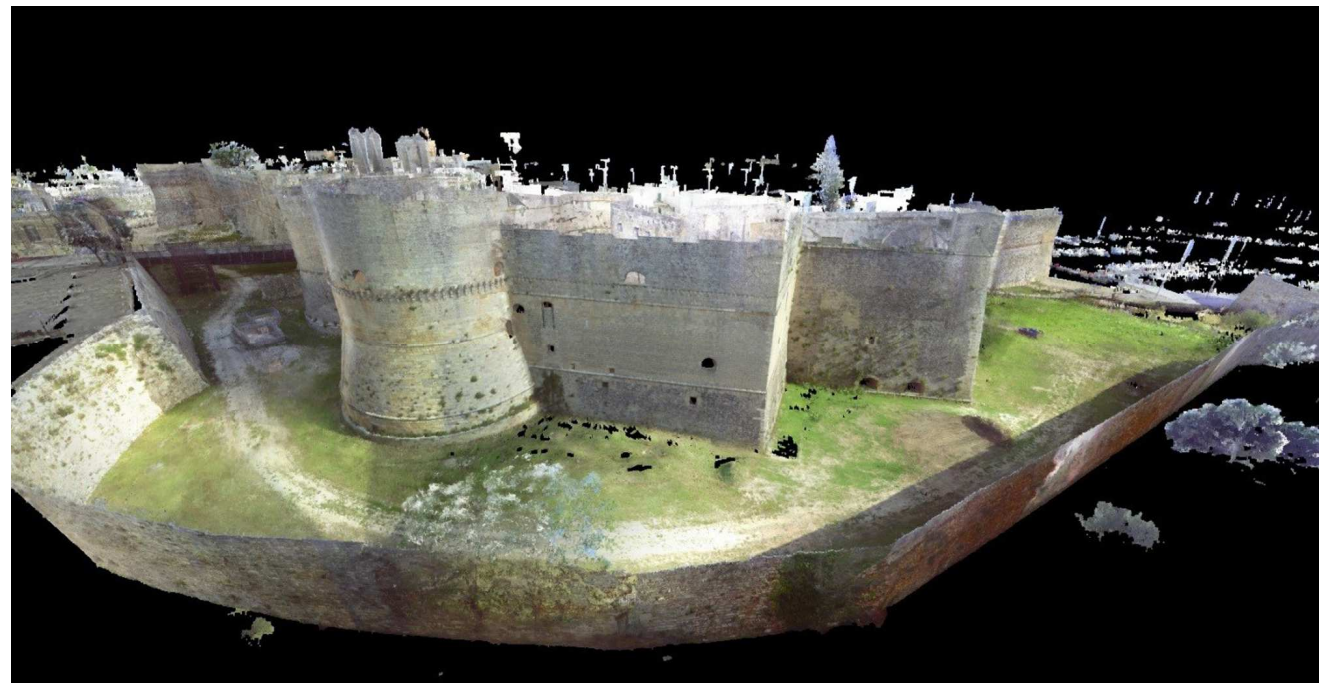

Fig. 4. Rilievo laser scanner 3D. Vista assonometrica del fossato e del fronte bastionato del castello aragonese. Il modello tridimensionale generato dalla nuvola di punti globale rappresenta lo stato di fatto del monumento con tutte le peculiarità architettoniche e costruttive (Mitello \& Muscatello, 2018). 

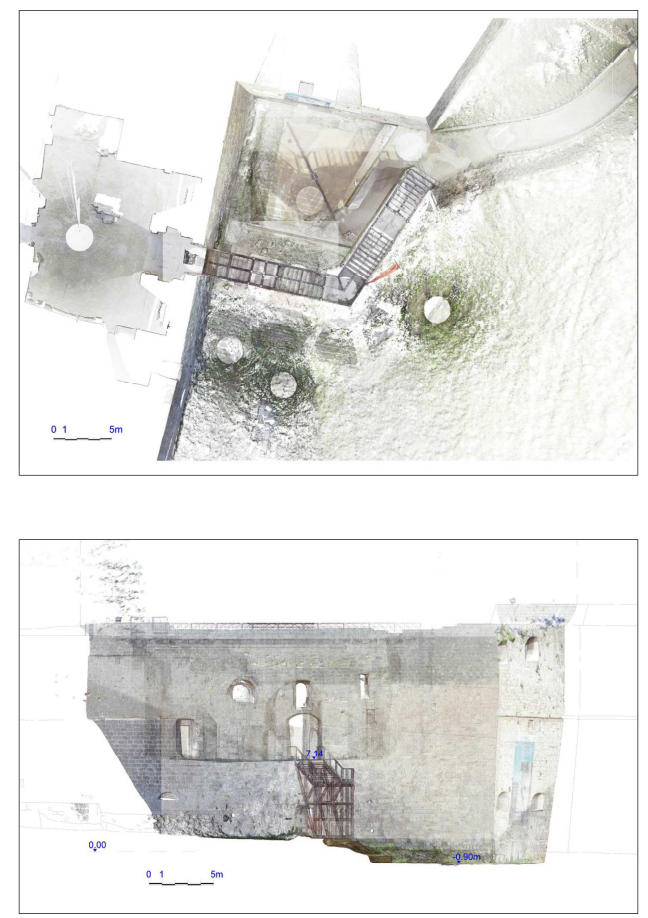

Fig. 5. Restituzione di prospetti e sezioni. Particolari.

dall'abitato, a cui era congiunto solo da un ponte levatoio, oggi di pietra. Non si hanno notizie certe relativamente al fossato medievale, celato probabilmente dagli interventi attuati dopo la presa della città idruntina da parte dei turchi nel 1480 , e dall' enorme quantità di materiale di interro che copre il piano di frequentazione originario del fossato che non consente la lettura di fasi più antiche. La configurazione attuale del percorso fortificato, caratterizzato dal castello e dalle rondelle dalla forma troncoconica con toro marcapiano è il risultato degli imponenti interventi di ricostruzione attuati dagli Aragonesi, dalla fine del ' 400 fino alla fine del ' 500 , che ripristinarono il circuito murario, inglobando le strutture esistenti e potenziando il sistema di difesa anche attraverso la definizione di un nuovo fossato per impedire le incursioni esterne. Il fossato si sviluppa per circa $1 \mathrm{~km}$ e corre accanto al perimetro delle alte mura e del fortilizio, da Porta Alfonsina (ad ovest) sino a Porta Terra, vicina alla Torre Matta, in prossimità dell'area portuale. Alcuni tratti del fossato sono interrotti da setti murari, soprattutto a ridosso del castello, edifica-
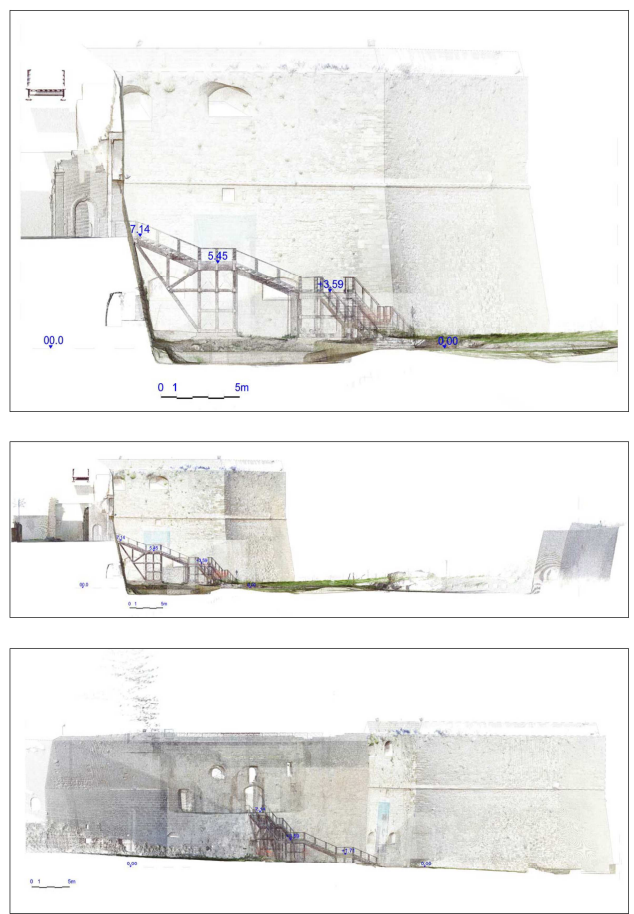

ti con elementi di riuso; creano settori isolati raggiungibili attraverso piccoli vani di accesso o percorrendo camminamenti interni alle mura, posti alla base della scarpata, alla quota dei fossati. E' particolarmente evidente l'ampiezza del fossato, scavato nel banco roccioso, che raggiunge distanze che vanno dai 15 ai 30 metri circa. Un sistema militare ben concepito che ha risposto alle nuove esigenze di difesa dopo la devastazione ottomana e conserva ancora testimonianze di attività antropiche più antiche, che sono ancora oggetto di indagine. E' plausibile che in parte ricalchi un più antico circuito murario vista la presenza, dietro la fodera aragonese, di enormi blocchi parallelepipedi allettati su piani orizzontali che rispecchiano la tecnica costruttiva del periodo messapico (V secolo a.C.).

\subsection{La cinta muraria: la Torre demolita}

Il rilievo e l'analisi delle murature in elevato hanno evidenziato alcune anomalie che interrompono la regolare tessitura della cortina aragonese. Un'attenta lettura stratigrafica ha permesso la registrazione di quelle azioni che hanno 
pesantemente segnato l'apparato murario. A ridosso della Torre denominata della "Duchesca" (fine XV secolo) è stato basilare l'esame dell'enorme impronta di una torre che s'innestava nell'originario perimetro difensivo, la cui demolizione ha lasciato una profonda traccia che consente una lettura capillare della forma e delle peculiarità costruttive (Fig. 6).

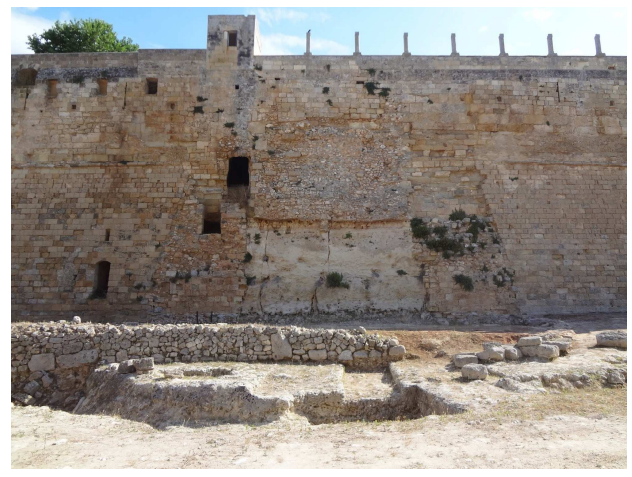

Fig. 6. Traccia della Torre demolita (Muscatello, 2017).

Alla base, a seguito della pulizia del fossato, è stata messa in luce la fondazione della costruzione ed è stato intercettato il profilo della muratura originaria ad andamento curvilineo.

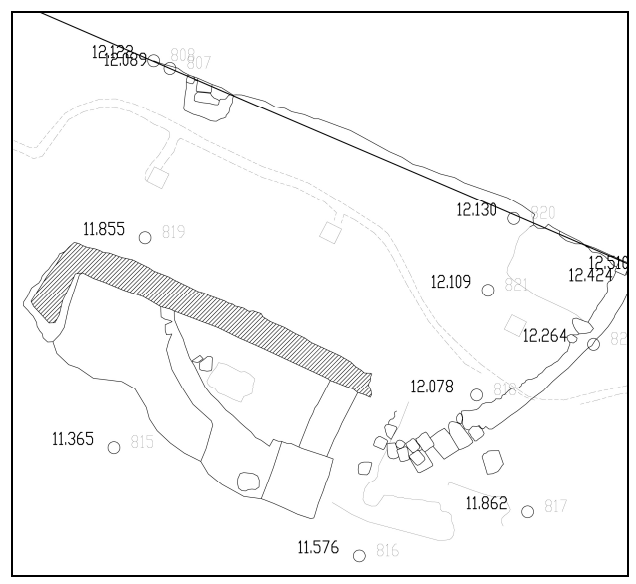

Fig. 7. Torre demolita. Rilievo plano-altimetrico del basamento e dei resti della muratura originaria (Mitello \& Muscatello, 2018).

Sul basamento ricavato nel banco calcarenitico sono ancora posati in giacitura primaria i primi blocchi di calcarenite, sagomati a forma di parallelepipedo, allettati su uno strato di malta con l'inclusione di inerti. Sparsi invece si ritrovano alcuni blocchi squadrati appartenenti alla cortina originaria della torre, taluni reimpiegati nella costruzione di un muretto a secco che taglia trasversalmente la fondazione e che probabilmente fu fabbricato nel XIX secolo come muro di terrazzamento quando l'area dei fossati venne utilizzata per scopi agricoli (Fig. 7). La conformazione planimetrica della torre demolita, dalla forma allungata e di dimensioni imponenti, differisce da quella emiciclica delle altre torri come la vicina Torre della Duchesca (Figg. 8 e 9).

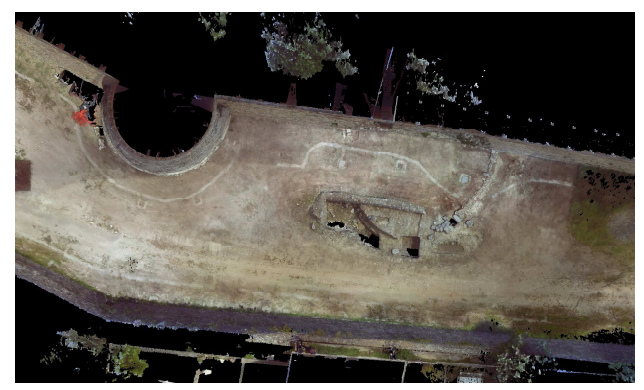

Fig. 8. Ortofoto da rilievo laser scanner 3D a colori della Torre della Duchesca (a sinistra) e del basamento della Torre demolita (a destra). In evidenza la differente conformazione planimetrica delle due torri (Mitello \& Muscatello, 2018).

La sezione visibile mette in risalto il nucleo roccioso intorno al quale si avvolgeva l'intera costruzione. La muratura era ben costituita con pietre di varia pezzatura e bolo in cui sono visibili i piani d'attesa. All'esterno era caratterizzata da un alto basamento e da un profilo a scarpa. Durante le operazioni di smontaggio del muro a secco, il ritrovamento di un pezzo modanato con una gola rovescia chiarisce la forma del toro marcapiano che cingeva la torre. All'interno della spessa muratura perimetrale si articolavano i percorsi che collegavano i piani e che plausibilmente conducevano alle bocche di fuoco distribuite nei vari ordini casamattati e che, nella parte compresa nelle mura della fortificazione, sono ancora percorribili. L'area in cui insiste il basamento della fondazione è stata già oggetto di indagini archeologiche preventive negli anni 


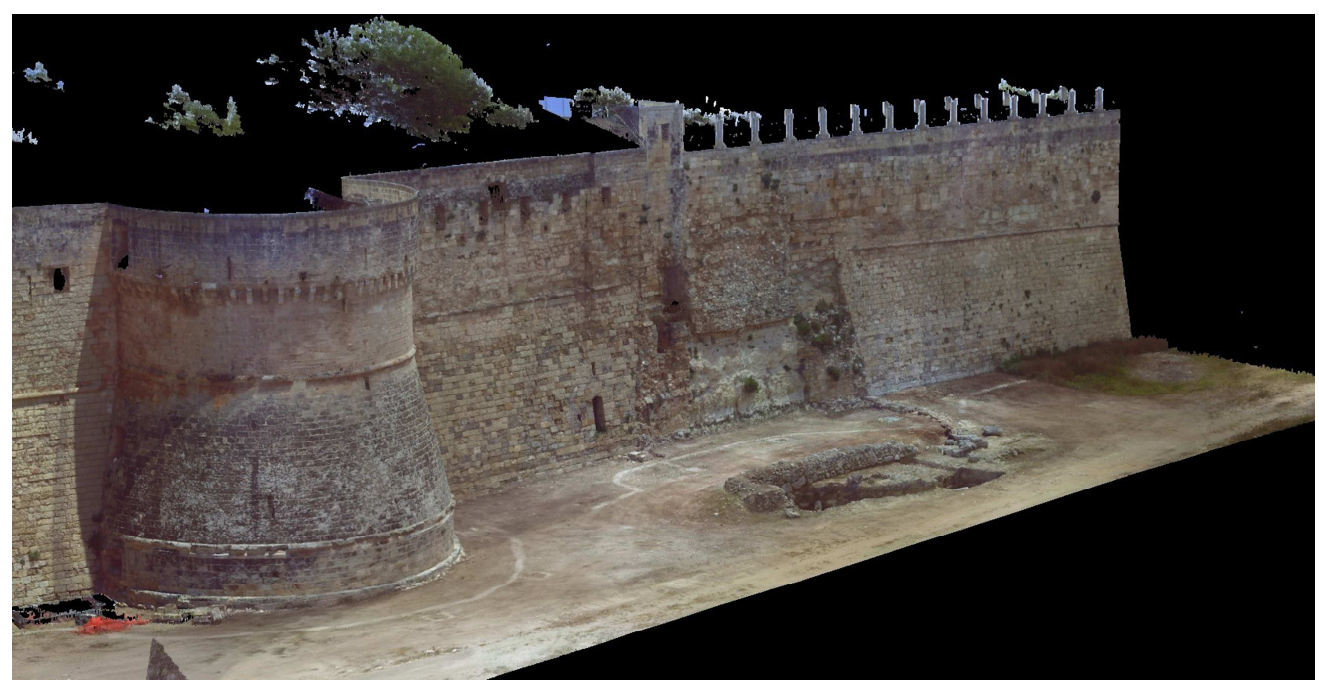

Fig. 9. Cinta muraria. Rilievo laser scanner 3D. Vista assonometrica della Torre della Duchesca (a sinistra) e della traccia della Torre demolita (a destra). In evidenza il paramento murario della cortina aragonese e la conformazione della rondella a pianta circolare con profilo a scarpa e toro marcapiano (Mitello \& Muscatello, 2018).

'90 del secolo scorso, nell'ambito degli interventi di svuotamento del fossato da tutto il materiale di accumulo che era stato riversato all'interno a partire dalla fine XIX secolo. Prima dello svuotamento la traccia della torre era solo parzialmente visibile, poiché il livello di interro arrivava sino alla postierla posta in alto a sinistra, a circa $10 \mathrm{~m}$ dall'odierno piano di calpestio. Probabilmente la torre è precedente alla contigua Torre della Duchesca e la sua demolizione fu eseguita nel momento in cui perse la funzione originaria, stante la ridefinizione dell'intera fortificazione idruntina alla fine del XV secolo

\subsection{Gli ambienti ipogei nel fossato}

A ridosso del castello e del ponte di legno, che permette l'accesso alla città antica passando sui fossati, sono presenti alcuni ambienti ipogei che rappresentano, per il momento, l'unica testimonianza di camminamenti sotterranei all'interno dei fossati (Figg. 10-12). Prima dell'intervento di pulizia dell'area nulla si vedeva di tali contesti, tranne le ringhiere in ferro che mettono in sicurezza le aree sottoposte. La vegetazione infestante ed i materiali di scarto di ogni tipo avevano celato quest'importante evidenza architettonica trasformandola in una discarica, rendendola impraticabile. L'opera di svuotamento ha restituito ambienti ipogei che presumibilmente vanno messi in relazione con il sistema dei camminamenti sotterranei articolati all'interno del castello. Nella parte sommitale sono evidenti i segni di cavatura, con i piani e linee di estrazione di blocchi lapidei. Il cunicolo principale è stato interamente scavato nella roccia calcarea. Si tratta di un'ambiente tecnicamente ben costruito dove sono visibili i segni di lavorazione. È voltato ed è alto circa $1,50 \mathrm{~m}$; il piano di calpestio si trova a circa 3 metri di profondità rispetto alla quota dei fossati. Al suo interno sono ubicati un pozzo a sezione rettangolare, ancora colmo di terra, e un vano più ampio sui cui lati lunghi sono visibili i piani di imposta di una volta a botte che originariamente lo copriva. Lungo le pareti della cisterna e del pozzo sono presenti degli incavi, le cosiddette "pedarole", praticati sulle pareti verticali e poste a distanza regolare per consentire e facilitare la discesa e la risalita. A sud-est il cunicolo è cieco e termina con il banco roccioso alla cui base è presente un sedile sagomato. A nord-ovest il passaggio è ostruito da terra e pietre di risulta, ma sembra continuare curvando verso il fronte principale del castello; non è da escludere che si articolasse intorno allo stesso a 
scopo difensivo, a protezione della parte più vulnerabile del maniero. E' plausibile l'ipotesi che tale opera sia una galleria di contromina, costruita nel periodo rinascimentale nell'ambito del potenziamento della fortezza e di tutto il sistema difensivo, con lo scopo di monitorare e contrastare lo scavo di eventuali gallerie da parte di minatori nemici in caso di assedio.

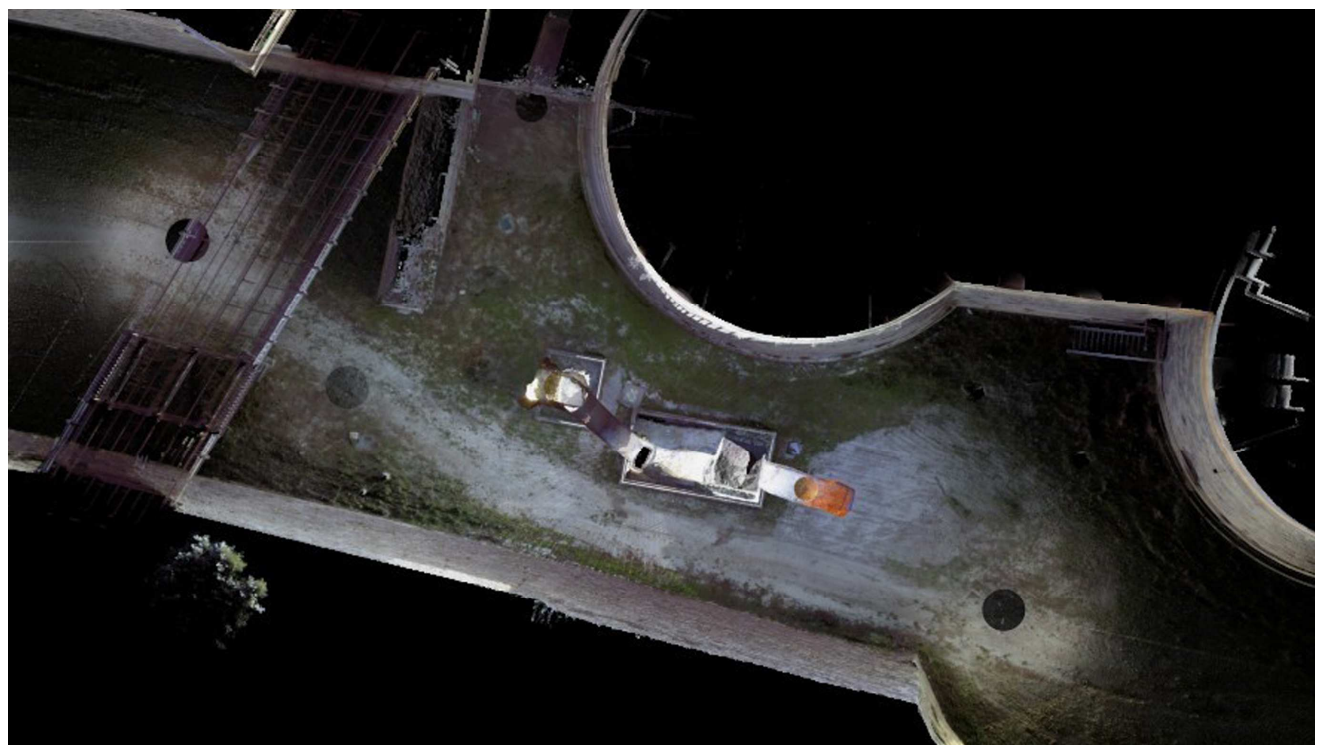

Fig. 10. Vista zenitale degli ambienti ipogei presenti nel fossato, a ridosso del torrione del castello e del ponte di legno (Mitello \& Muscatello, 2018).

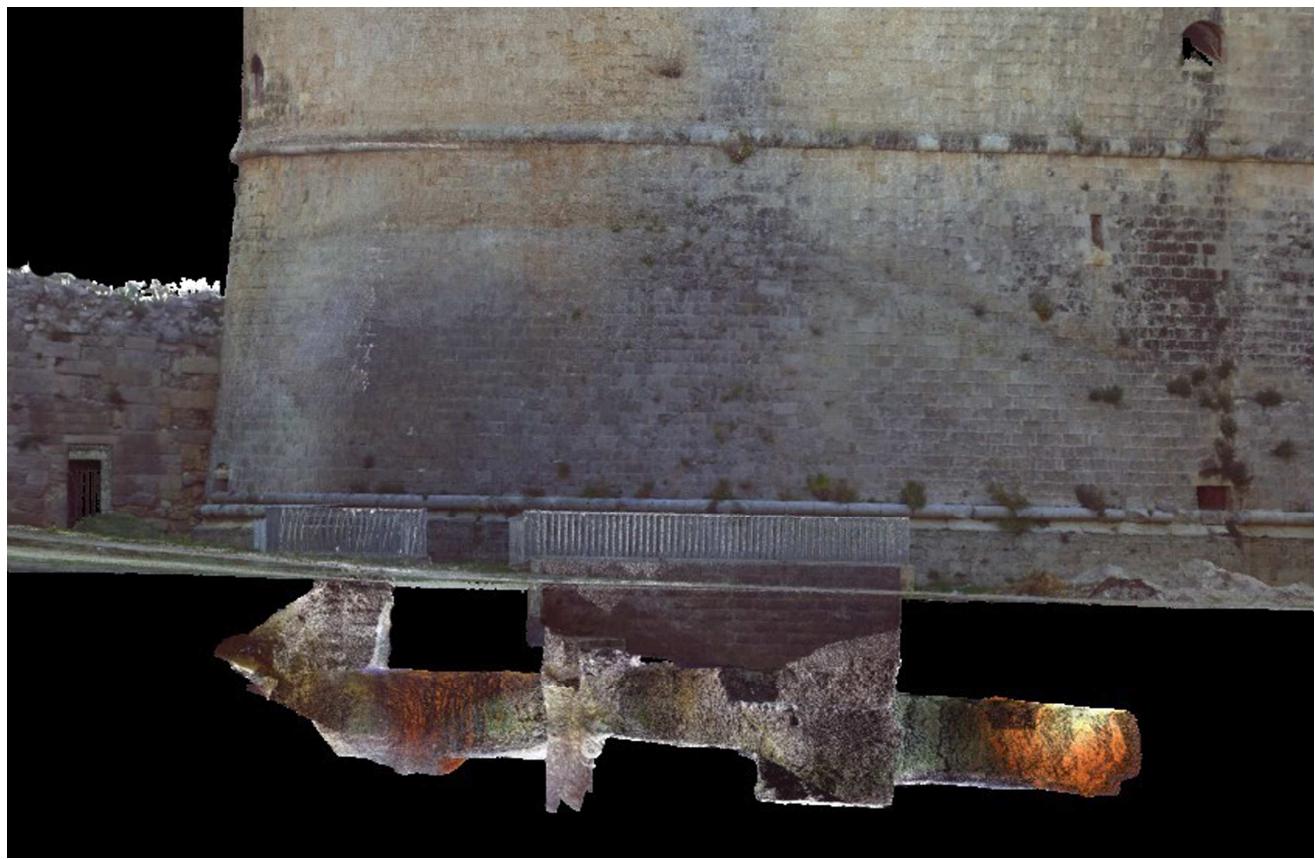

Fig. 11. Ambienti ipogei. Sezione da rilievo laser scanner 3D (Mitello \& Muscatello, 2018). 


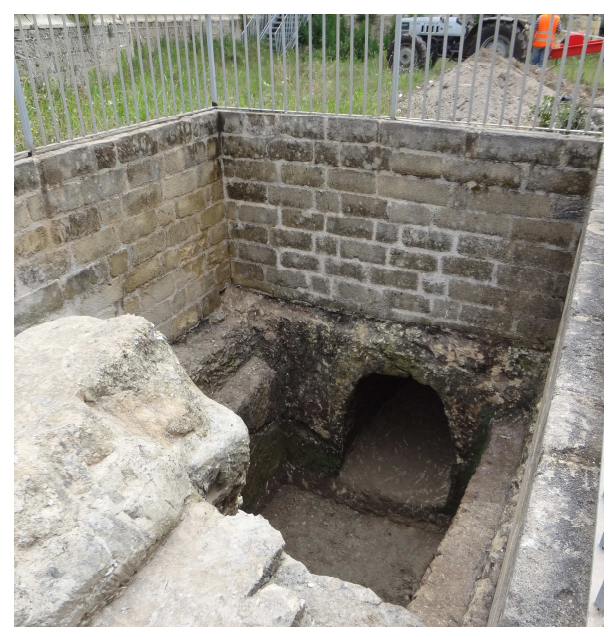

Fig. 12. Ambienti ipogei.

\section{Conclusioni}

L'analisi e il rilievo tridimensionale integrato delle evidenze architettoniche e archeologiche, alcune inedite, aggiungono un altro tassello alla comprensione del contesto monumentale preso in esame. La possibilità di studiare il modello tridimensionale nella sua globalità ha consentito di rivalutare alcune ipotesi pregresse a favore di nuove considerazioni in merito all'evoluzione del sistema fortificato della città di Otranto. La nuvola di punti globale elaborata costituisce un vasto archivio digitale da esplorare in funzione di nuovi percorsi di ricerca e ai fini di una corretta conservazione e tutela del complesso fortificato.

\section{Note}

${ }^{1}$ La ricerca è condotta nell' ambito del Progetto di "Recupero dei Fossati e la rifunzionalizzazione di una sala del castello da destinare a teatro virtuale" - Fondo per lo Sviluppo e Coesione 2007-2013.

Il rilievo con laser scanner 3D e le elaborazioni grafiche e tridimensionali sono stati realizzati da Carmine Mitello e da Giovanna Muscatello. Le immagini inserite nel testo sono di proprietà degli autori.

\section{Bibliography}

Bianchini, C. (2008). Manuale di rilievo e di documentazione digitale in archeologia, Aracne, Roma.

Cardaci, A.; Versaci, A. (2012). "Documentare per conservare: l'uso delle nuove tecnologie per la conoscenza, la salvaguardia e la comunicazione del patrimonio culturale armeno", Di-segnarecon, 5, 10, pp. 255-264, in http://disegnarecon.unibo.it/article/view/3354/2729.

Cariddi, P. (2014). Otranto intra moenia, dagli Aragonesi ad oggi, Esperidi Ed., Monteroni di Lecce.

Francovich, R.; Campana, S. (2006). Laser scanner e gps: paesaggi archeologici e tecnologie digitali, All'Insegna del Giglio, Firenze.

Muscatello, G.; Quarta, A.; Mitello, C. (2018). "I Sotterranei dei castelli di Otranto e Gallipoli: dal rilievo laser scanner 3D all'analisi strutturale", in: Marotta, A.; Spallone, R., eds., FORTMED 2018. Defensive Architecture of the Mediterranean, Politecnico di Torino Ed., Torino, vol. 9, pp.1021-1028.

Russo, M.; Remondino, F.; Guidi, G. (2011). "Principali tecniche e strumenti per il rilievo tridi-mensionale in ambito archeologico", Archeologia e Calcolatori, 22, pp. 169-198.

Vita, R. De. (1984). Castelli, torri ed opere fortificate di Puglia, Adda Ed., Bari. 